\title{
Robust modular control system design using an inner-loop reference model and $\mu$ synthesis techniques
}

\author{
Chris Vermillion ${ }^{1, *, \dagger}$, Jing $\operatorname{Sun}^{3}$ and Ken Butts ${ }^{2}$ \\ ${ }^{1}$ Altaeros Energies, Boston, MA 02210, USA \\ ${ }^{2}$ Toyota Technical Center, Ann Arbor, MI 48105, USA \\ ${ }^{3}$ Department of Naval Architecture and Marine Engineering, University of Michigan, Ann Arbor, MI 48109, USA
}

\begin{abstract}
SUMMARY
For complex dynamic systems, a modular control design process is often employed, wherein the overall design is partitioned into smaller modules. This paper considers a particular inner-loop/outer-loop modular control strategy in which the designer of the outer-loop module does not know the specifics of the inner loop but instead possesses a reference model that captures the ideal inner-loop input-output behavior. In the first part of this paper, we establish analytical properties of the modular reference-model-based design. In the second part, we introduce a novel mechanism, referred to as the modular control error compensation, which mitigates the performance loss that arises when the inner-loop reference model is not matched. We propose an iterative algorithm, using $\mu$ synthesis, to design this compensator to reduce performance loss on the basis of two concrete worst-case performance metrics. The effectiveness of the modular control strategy with the modular control error compensation is demonstrated through experimental results on an automotive system. Copyright () 2012 John Wiley \& Sons, Ltd.
\end{abstract}

Received 4 January 2011; Revised 3 March 2012; Accepted 11 March 2012

KEY WORDS: robust control; decentralized control; automotive control

\section{INTRODUCTION}

To address the complexities of many modern industrial systems, modular control designs are commonly used, where the overall control system is partitioned into multiple modules, each of which addresses a subset of the overall system dynamics. A very common modular strategy is an innerloop/outer-loop strategy, where an outer-loop controller is designed to achieve overall system performance objectives whereas an inner-loop controller is used to allocate control inputs among individual actuators. This strategy has seen widespread application in the automotive $[1,2]$, aerospace [3-7], and marine [8-12] industries. Additionally, theoretical work on inner-loop/outerloop cascade control designs also exists in [13] and [14].

The particular inner-loop/outer-loop strategy and corresponding nomenclature for this paper are shown in Figure 1, where the ultimate objective is to drive the performance variable, $y \in \mathbb{R}$, to a given setpoint, $r \in \mathbb{R}$. The outer-loop controller computes a desired overall effect, $v_{\text {des }} \in \mathbb{R}$, whereas the inner-loop control allocation determines control inputs, $u \in \mathbb{R}^{p}$, for the actuators, to closely track $v_{\text {des. }} v$ is commonly referred to as the virtual control input (and will be in this work), which represents an overall force, moment, or other generalized effect that drives the dynamics of the system. The control strategy in Figure 1 is widely employed in the industry because of its practicality in addressing the plant dynamics and actuator dynamics through separate controllers, particularly when different vendors are responsible for different components. The modular control

*Correspondence to: Chris Vermillion, Altaeros Energies, Boston, MA 02210, USA.

†E-mail: chris.vermillion@altaerosenergies.com 


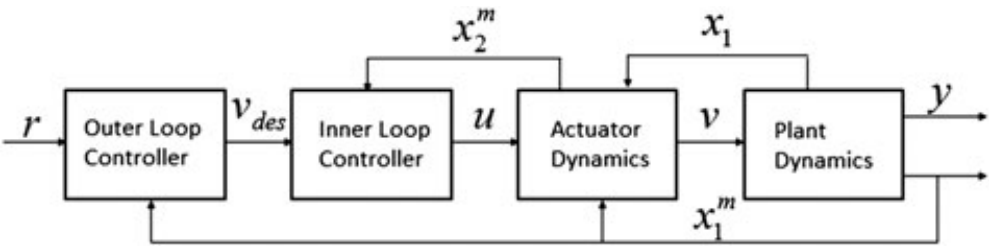

Figure 1. Block diagram of the overall system under a modular control strategy.

strategy splits the design work across different areas of expertise, allows plug-and-play application of different inner-loop modules, and reduces the complexity of the control allocation task.

When the actuator dynamics are very fast relative to the plant dynamics and overall closed-loop system requirements, the modular approach can easily be carried out by two designers in parallel. However, parallel design and seamless integration of the inner-loop and outer-loop controllers represent challenges when the inner-loop dynamics are significant relative to the outer-loop dynamics and exact tracking of $v_{\text {des }}$ is impossible. Different integration mechanisms have been proposed in the literature, each with its own benefits and pitfalls. For example, Luo et al. [7] and Tjonnas and Johansen [12] proposed algorithms that achieve asymptotic tracking of $v_{\text {des }}$ by performing a target state calculation at every instant and then designing the inner-loop controller to asymptotically track that target state trajectory. The ability to track $v_{\text {des }}$ asymptotically, however, requires advanced information about $v_{\text {des }}$ to be passed to the inner loop. These strategies, however, do not assess the implications of the delay, which can be significant in the presence of significant innerloop/outer-loop timescale separation, which must be introduced at the outer-loop level to provide sufficient advanced information to the inner loop. The strategies in [13] and [14] provide systematic design approaches for parallel outer-loop and inner-loop designs but also restrict the form that these controllers may take.

In this paper, we propose an alternative system integration approach to those proposed in earlier literature, which allows outer-loop and inner-loop designers to work in parallel. The proposed strategy does not place restrictions on the design methodology for either controller and does not require significant timescale separation between the inner and outer loops. In the proposed strategy, we introduce an inner loop reference model to capture the target inner-loop behavior from $v_{\text {des }}$ to $v$. This reference model can by tailored to reflect dynamics that are both desirable and realistic for the inner loop and can, under appropriate conditions, be matched exactly. In our analysis, we will derive, through a constructive proof, necessary and sufficient conditions on the reference model and actuator dynamics under which it is possible to match the reference model behavior. We also show that if the same conditions hold for the actuator dynamics, then the modular control strategy is capable of achieving equivalent performance to its centralized counterpart, shown in Figure 2. The ultimate result of this two-part analysis will be a set of ideal modular control conditions, which provide us with guidelines on how to choose the reference model and insight with regard to the features of the actuator dynamics that will cause difficulty in modular control design.

Because exact inner-loop model matching is not possible in many circumstances that involve uncertainties or nonideal properties of the actuator dynamics, the second part of this work proposes robust control techniques to manage this mismatch between the ideal and actual performance. Here,

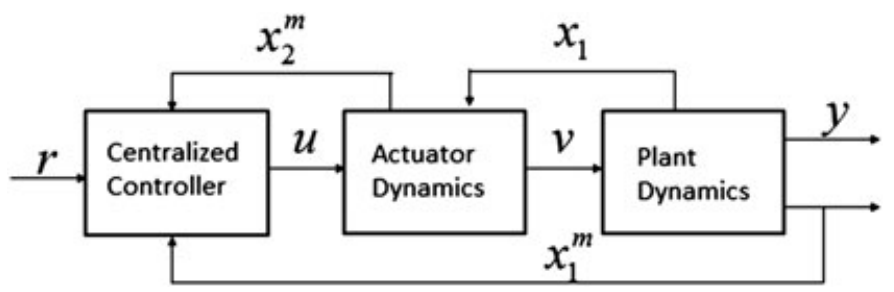

Figure 2. Block diagram of the overall system under a centralized control strategy. 
we capture the performance mismatch between the desired and actual inner closed loop through an uncertainty model and propose a novel control strategy, modular control error compensation (MCEC), to reduce the performance mismatch through use of an add-on compensator. This mechanism is inspired by the original work in [15], which considers a similar compensation mechanism but is restricted to parametric uncertainty. The MCEC design proposed in this work, which builds upon ideas from [15] and our earlier work in [16], uses $\mu$ synthesis for the compensator design. This results in a design framework that is applicable to a broader class of uncertainties and allows us to optimize performance on the basis of two concrete objectives. Furthermore, unlike other applications of $\mu$ synthesis such as in $[17,21]$, which impose an inherent trade-off between nominal performance and performance in the presence of uncertainty, our approach does not sacrifice nominal performance in achieving performance recovery in the presence of uncertainty. This is made possible by the fact that an add-on compensator, rather than the main outer-loop controller, is used to achieve performance recovery. We demonstrate the effectiveness of this strategy through experimental results on an automotive application example. Through this example, we also show how the usage and proper choice of an inner-loop reference model represents a key step in containing and ultimately reducing the mismatch between the ideal and actual system behavior.

The paper is organized as follows. In Section 2, we conduct an in-depth analysis of the referencemodel-based modular control design, deriving necessary and sufficient conditions for reference model matching and then demonstrating the close relationship between these conditions and a set of sufficient conditions for modular versus centralized equivalence. In Section 3, we treat the circumstances where the inner closed loop does not match the ideal behavior specified by the reference model. Here, we formally introduce MCEC and the uncertainty model that is used to describe the inner-loop performance mismatch. In Section 4 , we use this uncertainty model to formulate a $\mu$ synthesis problem to optimally design MCEC from two concrete worst-case performance metrics. Finally, in Section 5, we present design, analysis, and experimental results for modular control with MCEC on an automotive thermal management system.

\section{REFERENCE-MODEL-BASED MODULAR CONTROL DESIGN}

This section details the reference-model-based modular design framework, which is depicted in Figure 3. Mathematically speaking, the system in Figure 3 is identical to that in Figure 1; however, the block diagram has been manipulated in Figure 3 to illustrate the reference-model-based design process. Here, the inner-loop reference model, $F(s)$, represents the ideal performance from the desired virtual control input, $v_{\text {des }}$, to the virtual control input, $v . x_{1} \in \mathbb{R}^{n_{1}}$ represents the plant states, and $x_{2} \in \mathbb{R}^{n_{2}}$ represents the actuator states, whereas $x_{1}^{m}$ and $x_{2}^{m}$ represent those plant and actuator states, respectively, that are measured. $\tilde{v}$ represents the difference between the virtual control input, $v$, and the output of the reference model, $v_{\mathrm{des}}^{f}$. It is therefore the objective of the inner-loop controller to drive $\tilde{v}$ to 0 .

To characterize the performance achieved by the reference-model-based modular control design and quantify its design limitations, we will consider linear time-invariant systems whose dynamics are represented as follows:

$$
\begin{gathered}
X_{1}(s)=P^{x_{1}}(s) V(s), \\
Y(s)=P^{y}(s) V(s), \\
X_{2}(s)=A_{u}^{x_{2}}(s) U(s)+A_{x_{1}}^{x_{2}}(s) X_{1}(s), \\
V(s)=A_{u}^{v}(s) U(s)+A_{x_{1}}^{v}(s) X_{1}(s),
\end{gathered}
$$

where $P^{y}(s)$ is a scalar transfer function; $P^{x_{1}}(s), A_{u}^{v}(s)$, and $A_{x_{1}}^{v}$ are $n_{1} \times 1,1 \times p$, and $1 \times n_{1}$ transfer function vectors, respectively; and $A_{u}^{x_{2}}(s)$ and $A_{x_{1}}^{x_{2}}(s)$ are $n_{2} \times p$ and $n_{2} \times n_{1}$ transfer function matrices, respectively. The outer-loop and inner-loop control laws are given by

$$
V_{\mathrm{des}}(s)=C_{r}^{o}(s) R(s)+C_{x_{1}}^{o}(s) X_{1}(s)
$$




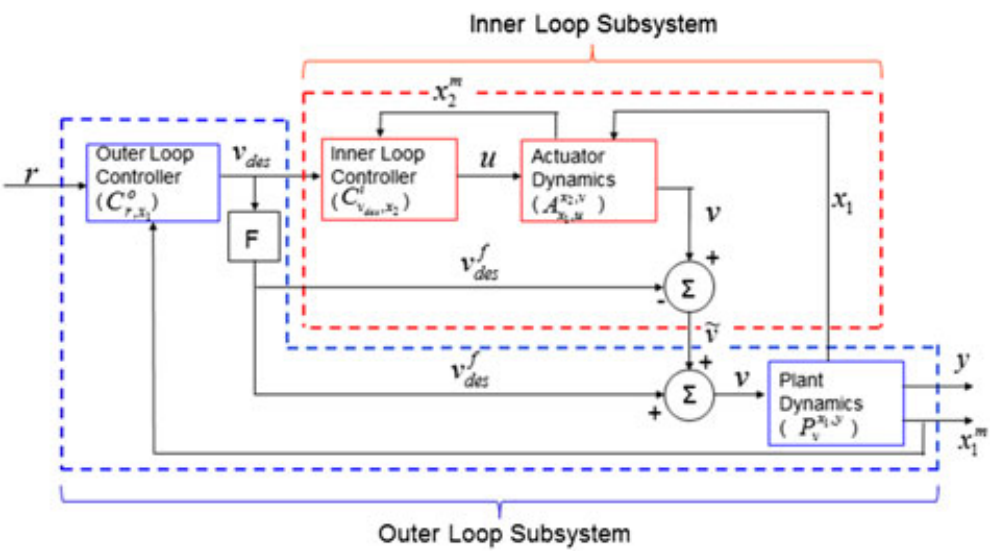

Figure 3. Block diagram of the reference-model-based modular control design.

and

$$
U(s)=C_{v_{\text {des }}}^{i}(s) V_{\text {des }}(s)+C_{x_{2}}^{i}(s) X_{2}(s),
$$

respectively, where $C_{r}^{o}(s)$ is a scalar transfer function, $C_{x_{1}}^{o}(s)$ and $C_{v_{\text {des }}}^{i}(s)$ are $1 \times n_{1}$ and $p \times 1$ transfer function vectors, respectively, and $C_{x_{2}}^{i}(s)$ is a $p \times n_{2}$ transfer function matrix.

\section{Remark 1}

In the absence of full state measurements, the entries in $C_{x_{1}}^{o}(s)$ and $C_{x_{2}}^{i}(s)$ corresponding to unmeasured states will be equal to 0 . Thus, we will typically omit the $x_{1}^{m}$ and $x_{2}^{m}$ notations throughout the paper, without loss of generality, to simplify the presentation.

Given an inner-loop controller $\left(C_{v_{\text {des }}}^{i}(s)\right.$ and $\left.C_{x_{2}}^{i}(s)\right)$, the closed-inner-loop input-output dynamics can be described by

$$
V(s)=T_{1}(s) V_{\text {des }}(s)+T_{2}(s) X_{1}(s),
$$

where, suppressing the $s$ notation for simplicity, we have

$$
\begin{gathered}
T_{1}=A_{u}^{v}\left(I-C_{x_{2}}^{i} A_{u}^{x_{2}}\right)^{-1} C_{v_{\mathrm{des}}}^{i}, \\
T_{2}=A_{u}^{v}\left(I-C_{x_{2}}^{i} A_{u}^{x_{2}}\right)^{-1}\left(C_{x_{2}}^{i} A_{x_{1}}^{x_{2}}\right)+A_{x_{1}}^{v} .
\end{gathered}
$$

For the modular reference-model-based control strategy, when the inner-loop reference model is matched, we have

$$
\begin{gathered}
T_{1}(s)=F(s), \\
T_{2}(s)=0,
\end{gathered}
$$

which we will refer to as the nominal closed inner loop. The notion of relative degree will be important to our subsequent analysis, and we will use the following scalar definition of relative degree.

\section{Definition 2}

A $1 \times q$ transfer function vector, $H(s)$, has relative degree $\rho(H(s)) \triangleq \min _{i} \rho\left(H_{i}(s)\right)$, where, taking $H_{i}(s)=N(s) / D(s), \rho\left(H_{i}(s)\right) \triangleq \operatorname{deg}(D(s))-\operatorname{deg}(N(s))$.

Thus, we take the relative degree of transfer function vectors as the lowest among the inputoutput pairs. 


\subsection{Overall system performance metric}

We will use the following metric for evaluating the overall system performance.

Definition 3 (overall performance index)

Let $G^{y}(s)$ and $G^{\text {des }}(s)$ represent the actual and desired transfer functions, respectively, from $r$ to $y$, and let $G^{v}(s)$ represent the actual transfer function from $r$ to $v$, and assume that these transfer functions are stable. The performance level, $\gamma^{p}$, of the closed-loop system is defined as

$$
\gamma^{p} \triangleq\left\|\left[W^{y}(s)\left(G^{y}(s)-G^{\operatorname{des}}(s)\right) \quad W^{v}(s) G^{v}(s)\right]\right\|_{\infty},
$$

where $W^{y}(s)$ and $W^{v}(s)$ are stable weighting transfer functions that emphasize or penalize performance at different frequencies.

This performance metric places importance on both setpoint tracking and the size of the virtual control input that is to be generated by the inner loop. In this section, we consider this performance on the nominal system, in the absence of uncertainty; however, subsequent sections treat the problem of recovering this nominal performance, to the greatest extent possible, in the presence of nonparametric uncertainty in the inner-loop dynamics.

\subsection{Inner-loop performance metric}

Because the objective of the inner-loop controller is to achieve $T_{1}(s)=F(s)$ and $T_{2}(s)=0$, the performance of the closed inner loop can be evaluated by

$$
\begin{gathered}
C^{i}=\arg \min _{C_{v_{\mathrm{des}}, x_{2}}^{i}} \gamma^{i}, \\
\gamma^{i}=\left\|\left[W^{1}(s)\left(F(s)-T_{1}(s)\right) W^{2}(s) T_{2}(s)\right]\right\|_{\infty} .
\end{gathered}
$$

Although we do not place any restrictions on the inner-loop control structure, we do derive a modelmatching controller for cases when $\gamma^{i}=0$ is achievable (through a constructive model-matching proof). We also derive both necessary and sufficient conditions for model matching to be achievable.

\subsection{Conditions for reference model matching}

As a first step of our analysis, we determine the conditions under which reference model matching, that is, $\gamma^{i}=0$, can be achieved.

\section{Proposition 4 (model-matching conditions)}

Suppose that the states $x_{2}$ are available for measurement, that is, $x_{2}^{m}=x_{2}$. Then, given an arbitrary, stable $F(s)$, there exists a causal and stabilizing inner-loop controller (i.e., proper and stabilizing transfer function matrices $C_{v_{\text {des }}}^{i}$ and $C_{x_{2}}^{i}$ ) that results in $V(s)=F(s) V_{\text {des }}(s)$ if and only if the following conditions exist:

1. $\rho\left(A_{u}^{v}(s)\right) \leqslant \rho(F(s))$.

2. $\rho\left(A_{u}^{v}(s)\right)<\rho\left(A_{x_{1}}^{v}(s)\right)$.

3. There are no unstable zeros common to all $p$ transfer functions in $A_{u}^{v}(s)$.

The detailed proof is provided in the Appendix. The proof of sufficiency involves construction of a controller that achieves model matching and stability. In particular, although a single control input is sufficient to achieve model matching, the proof shows that additional control inputs (if $p \geqslant 2$ ) can be used to stabilize the inner loop in the presence of nonminimum phase zeros. The necessity proof is quite straightforward and makes use of the notions of relative degree and stable pole-zero cancelations.

Because the reference model, $F(s)$, is a design choice, condition (1) in Proposition 4 can always be achieved and therefore does not restrict the set of systems for which model matching can be 
achieved. Condition (2) essentially requires the disturbance from $x_{1}$ to affect $v$ more slowly than $u$, such that state feedback can be used to reject this disturbance. In practice, there exist plants where this scenario is violated, and it is important, therefore, to address scenarios where system features or uncertainties lead to a mismatch between the reference model and actual inner-loop behavior (i.e., where $F(s) \neq T_{1}(s)$ or $T_{2}(s) \neq 0$ ). For this reason, Sections 3 and 4 of this paper are dedicated to performance recovery in these instances. Finally, condition (3) ensures that reference model matching can be achieved without introducing internal instability into the inner loop. The assumption degenerates into a very standard nonminimum phase model reference control assumption for a single-input, single-output inner loop, but multiple actuators provide greater flexibility in relaxing this condition.

\subsection{Modular versus centralized performance}

In this section, we compare the performance level of the modular control strategy with that of its centralized counterpart. For our performance comparison, we introduce the following notation.

\section{Definition 5}

Let $\gamma_{o}^{p}, \gamma_{c}^{p}$, and $\gamma_{m}^{p}$ represent the values of $\gamma^{p}$, as defined in (12), achieved with a given outer-loop controller when $T_{1}=F$ and $T_{2}=0$ (i.e., ideal modular control), with centralized control and with modular control, respectively.

Our comparison of these three levels of performance is given in the following proposition.

Proposition 6 (modular versus centralized performance inequality)

Assume that the optimal levels of performance achievable (i.e., the lowest values of $\gamma^{p}$, as defined in Definition 3) by the ideal modular design, centralized control system, and modular control system, under causal and stabilizing controllers, are given by $\gamma_{o}^{*}, \gamma_{c}^{*}$, and $\gamma_{m}^{*}$, respectively, and suppose that $F(s)$ is stable, is a minimum phase, and satisfies $\rho(F(s)) \leqslant \rho\left(A_{u}^{v}(s)\right)$ and $\rho(F(s)) \leqslant \rho\left(A_{x_{1}}^{v}(s)\right)$. Then, $\gamma_{o}^{*} \leqslant \gamma_{c}^{*} \leqslant \gamma_{m}^{*}$.

Proof

$\gamma_{c}^{*} \leqslant \gamma_{m}^{*}$ : Because the modular control system is a special case of the centralized controller, $\gamma_{c}^{*} \leqslant \gamma_{m}^{*}$ follows immediately.

$\gamma_{o}^{*} \leqslant \gamma_{c}^{*}$ : Consider an optimal centralized control law given by

$$
U^{*}(s)=C_{r}^{c}(s) R(s)+C_{x_{1}}^{c}(s) X_{1}(s)+C_{x_{2}}^{c}(s) X_{2}(s),
$$

which yields $\gamma_{c}^{p}=\gamma_{c}^{*}$. The corresponding closed-loop system transfer functions, $G^{v}(s)$ and $G^{y}(s)$, that are relevant to the performance index of (12) can be expressed by (suppressing the $s$ notation)

$$
\begin{gathered}
G^{v}=\left(1-A_{u}^{v}\left(I-C_{x_{2}}^{c} A_{u}^{x_{2}}\right)^{-1}\left(C_{x_{1}}^{c}+C_{x_{2}}^{c} A_{x_{1}}^{x_{2}}\right) P^{x_{1}}\right. \\
\left.-A_{x_{1}}^{v} P^{x_{1}}\right)^{-1} A_{u}^{v}\left(I-C_{x_{2}}^{c} A_{u}^{x_{2}}\right)^{-1} C_{r}^{c}, \\
G^{y}=P^{y} G^{v} .
\end{gathered}
$$

For the outer-loop controller in the modular control system, apply the causal and stabilizing control law

$$
V_{\mathrm{des}}(s)=F^{-1}(s)\left(A_{u}^{v}(s) \hat{U}(s)+A_{x_{1}}^{v}(s) X_{1}(s)\right),
$$

where

$$
\hat{U}(s)=\left(I-C_{x_{2}}^{c} A_{u}^{x_{2}}\right)^{-1}\left(C_{r}^{c} R+\left(C_{x_{1}}^{c}+C_{x_{2}}^{c} A_{x_{1}}^{x_{2}}\right) X_{1}\right) .
$$


Note that $\hat{u}$ in (19) is identical to $u^{*}$ in (15) when $x_{2}$ is solved for in terms of $u$ and $x_{1}$. When ideal conditions are met (i.e., $V(s)=F(s) V_{\text {des }}(s)$ ), this yields

$$
\begin{gathered}
G^{v}=\left(1-A_{u}^{v}\left(I-C_{x_{2}}^{c} A_{u}^{x_{2}}\right)^{-1}\left(C_{x_{1}}^{c}+C_{x_{2}}^{c} A_{x_{1}}^{x_{2}}\right) P^{x_{1}}\right. \\
\left.-A_{x_{1}}^{v} P^{x_{1}}\right)^{-1} A_{u}^{v}\left(I-C_{x_{2}}^{c} A_{u}^{x_{2}}\right)^{-1} C_{r}^{c} \\
G^{y}=P^{y} G^{v},
\end{gathered}
$$

which is identical to (16) and (17), thereby yielding $\gamma_{o}^{p}=\gamma_{c}^{*}$. Thus, it follows that $\gamma_{o}^{*} \leqslant \gamma_{c}^{*}$ $\leqslant \gamma_{m}^{*}$.

This simple inequality yields more profound implications when combined with the earlier reference-model-matching conditions of Proposition 4, leading to the following proposition.

Proposition 7 (modular versus centralized equivalence conditions)

Suppose the states $x_{2}^{m}=x_{2}, F(s)$ is stable and a minimum phase, $\rho(F(s))=\rho\left(A_{u}^{v}(s)\right)$, $\rho\left(A_{u}^{v}(s)\right)<\rho\left(A_{x_{1}}^{v}(s)\right)$, and $A_{u}^{v}(s)$ has no unstable zeros common to all $p$ transfer functions. Then, $\gamma_{o}^{*}=\gamma_{c}^{*}=\gamma_{m}^{*}$.

Proof

Suppose $F(s), C_{r}^{o}(s)$, and $C_{x_{1}}^{o}(s)$ are chosen optimally to yield $\gamma_{o}=\gamma_{o}^{*}$. From Proposition 4, there exists a causal, stabilizing inner-loop controller (with transfer functions $C_{v_{\text {des }}}^{i}(s)$ and $C_{x_{2}}^{i}(s)$ ) that yields $V(s)=F(s) V_{\text {des }}(s)$, thus yielding $\gamma_{m}=\gamma_{o}=\gamma_{o}^{*}$. It then follows immediately from Proposition 6 that $\gamma_{m}^{*}=\gamma_{c}^{*}=\gamma_{o}^{*}$.

\subsection{Summary—ideal modular control conditions}

We now have conditions on the actuator dynamics and reference model that, when satisfied, simultaneously yield reference model matching and modular versus centralized equivalence (based on the performance index, $\gamma^{p}$, defined in Definition 3). These conditions are summarized as follows:

1. $F(s)$ is stable and a minimum phase.

2. $\rho\left(A_{u}^{v}(s)\right)<\rho\left(A_{x_{1}}^{v}(s)\right)$.

3. $\rho(F(s))=\rho\left(A_{u}^{v}(s)\right)$.

4. $A_{u}^{v}(s)$ has no unstable zeros common to all $p$ constituent transfer functions.

In addition to providing a set of conditions on the actuator dynamics for which modular versus centralized equivalence can be achieved, these conditions also provide clear guidelines as to how the reference model, $F(s)$, should be chosen. Conditions (1) and (3) can always be satisfied with an appropriately designed reference model, whereas conditions (2) and (4) are dependent on the actuator dynamics themselves. Recognizing that modular control remains an appealing strategy even when these ideal conditions are not exactly met and when uncertainties render it impossible to match $F(s)$ exactly, in the remainder of this paper, we focus on mechanisms that reduce the gap between ideal and actual modular control performance. Because the impact of uncertainty on the overall system depends on one's choice of $F(s)$, we will also focus on how $F(s)$ can be fine-tuned for a particular uncertainty model.

\section{PERFORMANCE RECOVERY FORMULATION}

Attributes of the actuator dynamics, in conjunction with inevitable modeling uncertainties, will typically result in a mismatch between ideal and actual closed-inner-loop performance. To perform a 
more general analysis of system performance in the presence of this mismatch, we first express the closed inner loop through an uncertainty model:

$$
\begin{aligned}
T_{1} & =F+W_{1} \Delta_{1}, \\
T_{2} & =W_{2} \Delta_{2}, \\
\left\|\Delta_{1}\right\|_{\infty} & \leqslant \Delta_{1}^{\max }, \\
\left\|\Delta_{2}\right\|_{\infty} & \leqslant \Delta_{2}^{\max } .
\end{aligned}
$$

$W_{1,2}$ are known stable transfer function matrices of size $1 \times 1$ and $1 \times n_{1}$, respectively, that capture information known about the mismatch between the actual and ideal inner-loop performance.

The design of weighting functions $W_{1,2}$ in (22) represents an important design decision that will affect the feasibility of the forthcoming $\mu$ synthesis design and the engineering value that such a design will have. We summarize general guidelines for the design of $W_{1,2}$ and then show in Section 5 how these guidelines are applied on an application example.

To ensure well-posedness of the $\mathrm{D}-\mathrm{K}$ iteration that is used in subsequent $\mu$ synthesis design, we impose the following requirement on $W_{1,2}$ :

There does not exist a frequency, $\omega \geqslant 0$, at which $W_{2}(j \omega)=0$ and $W_{2 i}(j \omega)=0, i=1 \ldots n$.

This requirement is not difficult to achieve and simply requires at least one weighting function to have a nonzero gain at any given frequency. Two engineering approaches can be used to choose a weighting function that is appropriate for the system at hand:

(1) Simulation of likely parametric uncertainty: Given some physical understanding of the system at hand and the simplifications made in the modeling approach, one can identify likely sources of parametric uncertainty. The designer can then generate Bode plots of $T_{1}-F$ and $T_{2}$ under random variations in these uncertain parameters and shape $W_{1}$ and $W_{2}$ to match the shapes of these Bode plots, subject to well-posedness restrictions on $W_{1,2}$.

(2) Experimental uncertainty characterization: Given sufficiently rich experimental data for the closed-inner-loop system and the frequency content of $v_{\text {des }}$ and $x_{1}$, one can also approximate the frequency domain behavior of $T_{1}-F$ and $T_{2}$ and shape $W_{1,2}$.

To quantify the difference between the output of the nominal and actual (with uncertainty) closed-loop systems, we introduce a new variable, $e^{\prime}$, which is defined as follows:

$$
e^{\prime} \triangleq y-y^{r} \text {. }
$$

Here, $y$ represents the actual output whereas $y^{r}$ represents the output when (10) and (11) are satisfied. For notational convenience and in preparation for later $\mu$ analysis and synthesis, we will cast both uncertainty sources $\left(\Delta_{1}\right.$ and $\left.\Delta_{2}\right)$ together in a block diagonal matrix, as follows:

$$
\Delta=\left[\begin{array}{cc}
\Delta_{1} & 0 \\
0 & \Delta_{2}
\end{array}\right] \text {. }
$$

Given this uncertainty representation, the closed-inner-loop error dynamics can be described by

$$
\tilde{V}=\left[\begin{array}{ll}
W_{1} & W_{2}
\end{array}\right]\left[\begin{array}{cc}
\Delta_{1} & 0 \\
0 & \Delta_{2}
\end{array}\right]\left[\begin{array}{c}
V_{\mathrm{des}} \\
X_{1}
\end{array}\right] .
$$

\section{Remark 8}

Although the uncertainty in (24) assumes a block diagonal structure, this structure is not restrictive in the sense that this structure allows every input to the inner closed loop $\left(v_{\text {des }}\right.$ and every element of $x_{1}$ ) to affect the single output, $v$, through an uncertain transfer function.

For performance recovery, we will make extensive use of the worst-case gain, $\gamma^{\mathrm{wc}}$, from $r$ to $e^{\prime}$, where $\gamma^{\mathrm{wc}}$ is defined as

$$
\gamma^{\mathrm{wc}} \triangleq \max _{\|\Delta\|_{\infty} \leqslant \Delta_{\max }}\left\|G^{e r}\right\|_{\infty}
$$


where

$$
G^{e r} \triangleq \frac{E^{\prime}(s)}{R(s)} .
$$

Estimation and analysis of worst-case performance in an uncertain system has been the topic of extensive literature (studied, for example, in [22]), and tools such as MATLAB's Robust Control Toolbox have facilitated the estimation of $\gamma^{\mathrm{wc}}$ for numerous robust control applications such as ours.

We propose a novel mechanism, referred to as MCEC, which is depicted in Figure 4 and is characterized by an add-on compensator, $C_{v}$. The proposed MCEC design structure exploits the signal $\tilde{v}$, which represents the difference between the virtual control input, $v$, and its nominal value, $v_{\text {des }}^{f}$ (the output of $F$ ), to reduce the gap between nominal and actual performance. The proposed strategy differs from other robust controller synthesis applications, such as in [17-20], which design the main controller to guarantee a certain level of overall system performance in the presence of uncertainty. Because the strategies take into consideration the nominal model as well as uncertainty in their design, they impose an inherent trade-off between nominal performance and performance in the presence of uncertainty. Our structure, employing $C_{v}$ as an add-on compensator, has two key advantages over strategies that redesign the main controller for robust performance:

(1) Our strategy retains the modularity of the inner and outer loops in the sense that it allows the two controllers to be designed in parallel without knowledge of each other;

(2) There is no trade-off between nominal performance and performance in the presence of uncertainty-when the inner-loop reference model is matched $\left(T_{1}(s)=F(s), T_{2}(s)=0\right)$, $\tilde{v}$ will go to 0 .

We show that by recasting the optimization problem for the MCEC design, we can formulate a $\mu$ synthesis problem to optimize specific worst-case performance on the basis of two synthesis objectives, $\mathrm{S} 1$ and $\mathrm{S} 2$ :

S1 Given that $\|\Delta\|_{\infty}<1$, find a stabilizing $C_{v}$ that minimizes $\gamma^{\mathrm{wc}}$.

S2 Given $\gamma^{\text {th }}>0$ ( $\gamma^{\text {th }}$ is a performance threshold representing tolerable performance degradation), find $C_{v}$ that maximizes $\|\Delta\|_{\infty}$ such that the closed-loop system remains stable and $\gamma^{\text {wc }}<\gamma^{\text {th }}$.

\section{MODULAR CONTROL ERROR COMPENSATION DESIGN FOR PERFORMANCE RECOVERY USING $\mu$ SYNTHESIS}

To design $C_{v}$, the block diagram in Figure 4 is cast in the form given by Figure 5, where $\bar{P}$ is a transfer function matrix containing all of the system components besides $C_{v}, \Delta$, and scalar gain $k$.

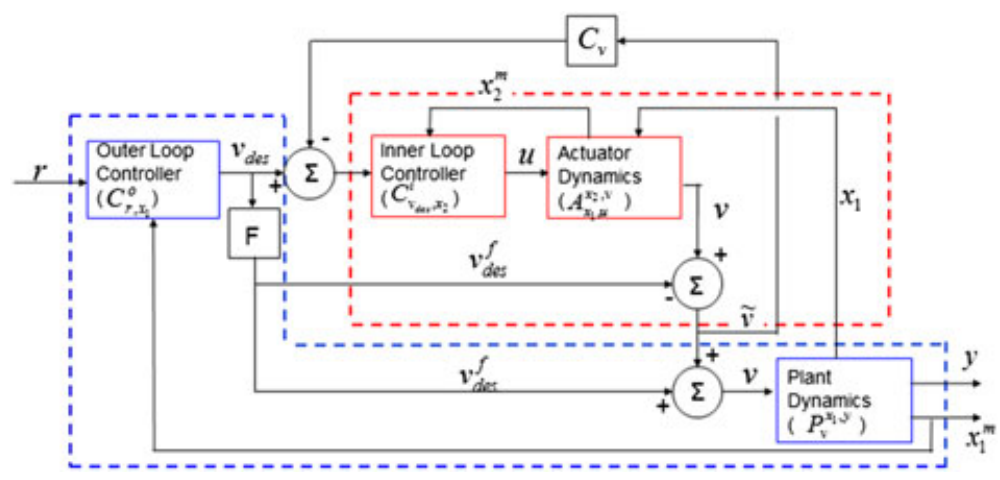

Figure 4. Block diagram of the reference-model-based modular control design with modular control error compensation. 


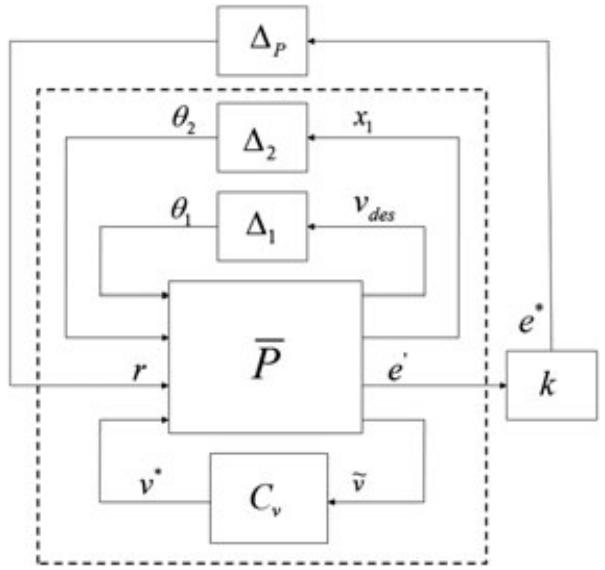

Figure 5. $\mu$ synthesis performance formulation. Physical entities of the system are contained inside the dashed lines, whereas fictitious entities $\left(k\right.$ and $\left.\Delta_{P}\right)$, which are strictly introduced for $\mu$ synthesis design purposes, lie outside.

The expression for $\bar{P}$ is given in (29). Under this configuration, $\mu$ synthesis considers an uncertainty structure given by

$$
\Delta_{\text {aug }} \triangleq\left[\begin{array}{cc}
\Delta & 0 \\
0 & \Delta_{P}
\end{array}\right]
$$

The following properties hold for the system in Figure 5 [23].

Proposition 9 (robust performance interpretation of $\mu$ )

Let $\mu$ be the structured singular value for the system shown in the figure of Balas [23]. The following two properties hold:

(1) The closed-loop system in Figure 5 is stable and $k\left\|G^{e r}\right\|_{\infty}<\mu$ for all uncertainties satisfying $\|\Delta\|_{\infty}<1 / \mu$.

(2) There exists a perturbation $\Delta:\|\Delta\|_{\infty}=1 / \mu$ for which the closed-loop system in Figure 5 is unstable or $k\left\|G^{e r}\right\|_{\infty}=\mu$.

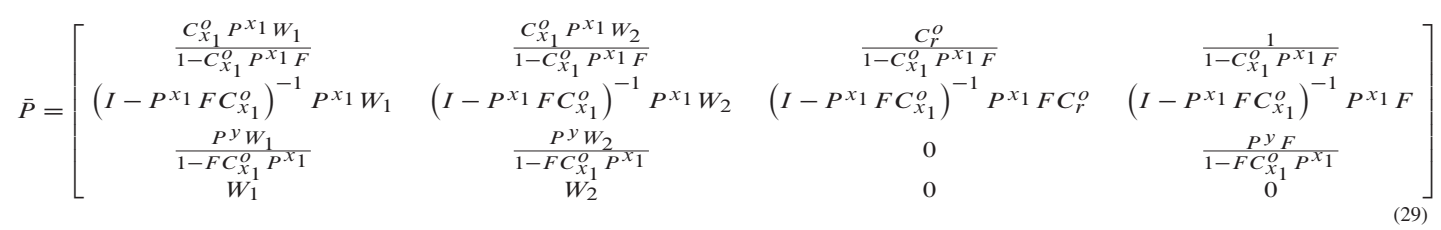

$\mu$ synthesis optimizes $C_{v}$ to minimize $\mu$, which allows us to carry out objectives $\mathrm{S} 1$ and $\mathrm{S} 2$. This optimization is carried out through the so-called D-K iteration [23], which relies on an underlying iterative $H_{\infty}$ optimization and is subject to the following well-posedness conditions:

(1) The last row of $\bar{P}(j \omega)$ is right invertible for all $\omega \geqslant 0$.

(2) The last column of $\bar{P}(j \omega)$ is left invertible for all $\omega \geqslant 0$.

Condition (1) can be satisfied through appropriate design of $W_{1,2}$, as detailed in Section 4. Condition (2) requires the gain of at least one of the transfer functions in the last column of $\bar{P}$ to be nonzero at any given frequency.

Given that the well-posedness conditions have been satisfied, the following propositions show how one can develop a design algorithm to achieve the synthesis objectives by iteratively adjusting $k$ and carrying out $\mu$ synthesis to design $C_{v}$. 
Proposition 10 (synthesis objective S1)

Assume that $\mathrm{S} 1$ is feasible. Let $\mu^{*}(k, \bar{P})$ be the structured singular value of the system shown in Figure 5 where $C_{v}$ has been designed using $\mu$ synthesis, for a given constant $k$. Furthermore, let $k_{1}^{*}$ be the maximum value of $k$ such that $\mu^{*}(k, \bar{P})=1$. Then, $k_{1}^{*}$ exists. Furthermore, if $C_{v}$ is designed using $\mu$ synthesis, with $k=k_{1}^{*}$, then $\gamma^{\mathrm{wc}}$ is minimized and the closed loop is stable, subject to $\|\Delta\|_{\infty}<1$.

\section{Proof}

Existence: Given that $\mathrm{S} 1$ is feasible, there exists a stabilizing $C_{v}$ when $\|\Delta\|_{\infty}<1$, which results in a finite $\gamma^{\mathrm{wc}}$. When $k=1 / \gamma^{\mathrm{wc}}$ is taken into account, it follows from Proposition 9 that $\mu\left(C_{v}, k, \bar{P}\right)=1$, thereby proving the existence.

When $C_{v}$ is designed with $k=k_{1}^{*}$, the closed loop is stable and $\gamma^{w c}$ is minimized, subject to $\|\Delta\|_{\infty}<1$ : Closed-loop stability when $\mu^{*}=1$ follows directly from property (1) of Proposition 9. To show that $\gamma^{\mathrm{wc}}$ is minimized, we first show by contradiction that $\gamma^{\mathrm{wc}}=\mu^{*} / k_{1}^{*}$. Suppose that $\gamma^{\mathrm{wc}}<\mu^{*} / k_{1}^{*}\left(\gamma^{\mathrm{wc}}>\mu^{*} / k_{1}^{*}\right.$ violates Proposition 9, property (1), and need not be considered). Then, there exists $k>k_{1}^{*}$ that also yields $\mu^{*}(k, \bar{P})=1$, which contradicts the fact that $k_{1}^{*}$ is the maximum value of $k$ yielding $\mu^{*}(k, \bar{P})=1$. Therefore, $\gamma^{\text {wc }}=\mu^{*} / k_{1}^{*}=1 / k_{1}^{*}$. Because $k$ is maximized and $\mu$ synthesis minimizes $\mu, \gamma^{\text {wc }}$ is minimized for all $C_{v}, k$ that yield $\mu\left(C_{v}, k, \bar{P}\right)=1$. Finally, note that for all $C_{v}$ that yield a stable closed loop when $\|\Delta\|_{\infty}<1$, there exists a $k$ such that $\mu\left(C_{v}, k, \bar{P}\right)=1$. Therefore, minimizing $\gamma^{\text {wc }}$ over all $C_{v}, k$ that yield $\mu\left(C_{v}, k, \bar{P}\right)=1$ is equivalent to minimizing $\gamma^{\mathrm{wc}}$ over all $C_{v}$ that yield a stable closed loop when $\|\Delta\|_{\infty}<1$.

\section{Proposition 11 (synthesis objective S2)}

Let $\mu^{*}(k, \bar{P})$ be the structured singular value of the system shown in Figure 5 where $C_{v}$ has been designed using $\mu$ synthesis, for a given constant $k$. Let $k_{2}^{*}$ be the minimum value of $k$ such that $\mu^{*}(k, \bar{P})=k \gamma^{\text {th }}$. Then $k_{2}^{*}$ exists, and if $C_{v}$ is designed with $k=k_{2}^{*}$, then $\mu$ synthesis maximizes the value of $\|\Delta\|_{\infty}$ such that $\gamma^{\mathrm{wc}}<\gamma^{\text {th }}$ and the closed-loop system remains stable.

The proof is omitted but follows exactly the same logical sequence as the proof of Proposition 10. Using Propositions 9 and 10, we propose the following iterative algorithm to design the MCEC controller $C_{v}$ to achieve objectives S1 and S2; for S1, take $\mu^{o}=1$, and for S2, take $\mu^{o}=k \gamma^{\text {th }}$ :

(1) (a) Initialize $k_{\text {low }}$ to any value that is known to satisfy $k_{\text {low }}<k_{i}^{*}$ ( $i=1$ or $i=2$ depending on the objective). Take $k_{\text {low }}=0$ if no other lower bound is known. Proceed to step (1b).

(b) Initialize $k_{\text {high }}$ to any value that is known to satisfy $k_{\text {high }}>k_{i}^{*}$, and proceed to step (2). If no upper bound on $k$ is known, make an initial guess, $k_{\text {init }}$, and carry out $\mu$ synthesis for $k=k_{\text {init }}$.

(i) If $\mu^{*}\left(k_{\text {init }}, \bar{P}\right)-\mu^{o}>\epsilon$, take $k_{\text {high }}=k_{\text {init }}$ and continue with step (2).

(ii) If $\mu^{*}\left(k_{\text {init }}, \bar{P}\right)-\mu^{o}<\epsilon$, increase $k_{\text {init }}$ and repeat step (1b).

(2) Carry out $\mu$ synthesis for $k=1 / 2\left(k_{\text {high }}+k_{\text {low }}\right)$, which will return $C_{v}$ and $\mu^{*}$. Proceed to step (3).

(3) (a) If $\left|\mu^{*}-\mu^{o}\right|<\epsilon$, move to step (4) for S1 and step (5) for S2.

(b) If $\mu^{*}-\mu^{o}<-\epsilon$, set $k_{\text {low }}=k$ and repeat step (2).

(c) If $\mu^{*}-\mu^{o}>\epsilon$, set $k_{\text {high }}=k$ and repeat step (2).

(4) (a) If $\left|k_{\text {high }}-k\right|<\epsilon_{k}$, where $\epsilon_{k}$ is a user-defined threshold, terminate the algorithm.

(b) If $\left|k_{\text {high }}-k\right| \geqslant \epsilon_{k}$, take $k_{\text {low }}=k$ and move to step (4c).

(c) Take $k=1 / 2\left(k_{\text {low }}+k_{\text {high }}\right)$, and carry out $\mu$ synthesis.

(i) If $\left|\mu^{*}(k, \bar{P})-\mu^{o}\right| \geqslant \epsilon$, set $k_{\text {high }}=k$ and repeat step (4c) until $\left|k_{\text {high }}-k\right|<\epsilon_{k}$.

(ii) Otherwise, take $k_{\text {low }}=k$ and repeat step (4c) until $\left|k_{\text {high }}-k\right|<\epsilon_{k}$ and then terminate.

(5) (a) If $\left|k-k_{\text {low }}\right|<\epsilon_{k}$, terminate the algorithm.

(b) If $\left|k-k_{\text {low }}\right| \geqslant \epsilon_{k}$, take $k_{\text {high }}=k$ and move to step (5c).

(c) Take $k=1 / 2\left(k_{\text {low }}+k_{\text {high }}\right)$, and carry out $\mu$ synthesis.

(i) If $\left|\mu^{*}(k, \bar{P})-\mu^{o}\right| \geqslant \epsilon$, set $k_{\text {low }}=k$ and repeat step (5c) until $\left|k-k_{\text {low }}\right|<\epsilon_{k}$.

(ii) Otherwise, take $k_{\text {high }}=k$ and repeat step (5c) until $\left|k-k_{\text {low }}\right|<\epsilon_{k}$ and then terminate. 


\section{Remark 12}

As an alternative to the iterative procedure proposed here, a skew $\mu$ synthesis procedure may be employed, in which the size of one uncertainty block is held fixed whereas the other(s) are allowed to vary $[24,25]$. This framework requires additional mathematical tools to set up but may result in reduced computational effort once implemented.

\section{ENGINE THERMAL MANAGEMENT APPLICATION}

The proposed modular control design approach is now studied on an engine thermal management system, which is used to facilitate engine mapping and calibration within an automotive test cell. The system, depicted in Figure 6, with a photograph of the actual experimental system given in Figure 7 (showing the unit containing the heater, heat exchanger, and mixing valve, which is connected through flexible piping to the engine), consists of parallel heating and cooling loops, used for controlling fluid temperature at the engine outlet. Readers are referred to [26] and [27] for system details, including a complete model description.

\subsection{Modular system description and properties}

The states in the thermal management system consist of eight temperatures, namely

- $T_{\text {out,eng }}=$ engine outlet temperature,

- $T_{\text {eng1 }}=$ engine block temperature in the vicinity of coolant,

- $T_{\text {eng2 }}=$ engine block temperature in the vicinity of oil,

- $T_{\text {out,mv }}=$ mixing valve outlet temperature,

- $T_{\text {outht }}=$ heater outlet temperature,

- $T_{\mathrm{ht}}=$ heater coil temperature,

- $T_{\text {outhex }}=$ heat exchanger outlet temperature,

- $T_{\text {out,cw }}=$ cooling water temperature at its outlet from the heat exchanger.

In this work, we utilize an effective general control strategy proposed in [27], where the heater is kept at a constant input of $2.25 \mathrm{~kW}$, which has been deemed optimal for the speed and load that we consider in our experiments $(2000 \mathrm{rpm}$ and $75 \mathrm{~N} / \mathrm{m})$. We perform a linearization about $T_{\text {out,eng }}=80^{\circ} \mathrm{C}$ (and the corresponding steady state), and the valve, with control input $\phi$, is manipulated to achieve temperature tracking. We work with translated coordinates, that is, $\delta T_{\text {out,eng }}=$ $T_{\text {out,eng }}-T_{\text {out,eng }}^{\text {ss }}$, etc., as well as a translated control input, $\delta \phi$, with the ultimate objective of controlling engine outlet temperature ( $T_{\text {out,eng }}$ ) to a setpoint. The virtual control input is taken as $T_{\text {out,mv }}$,

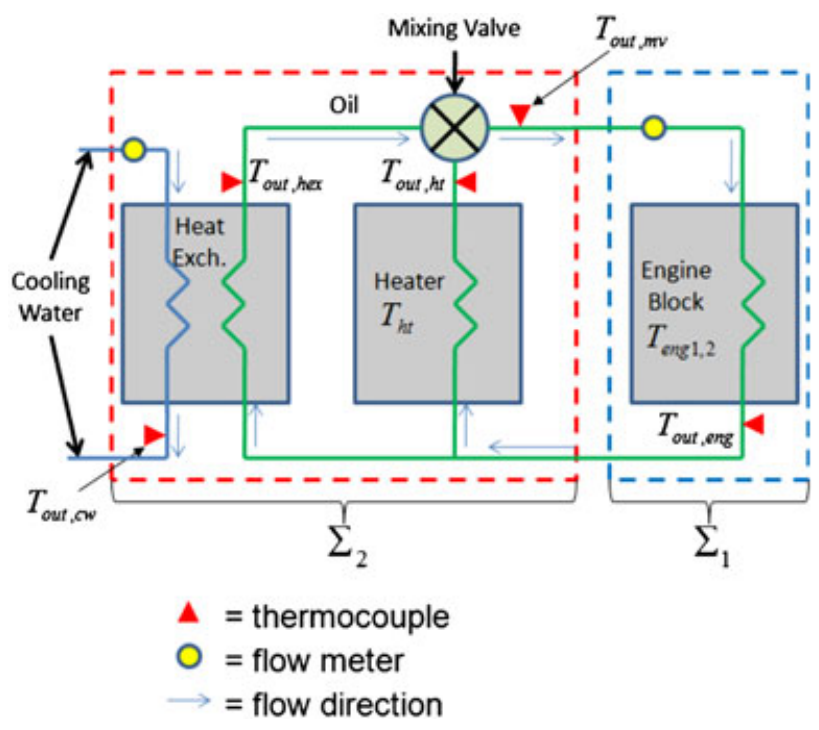

Figure 6. Thermal management system diagram (for the oil loop). 


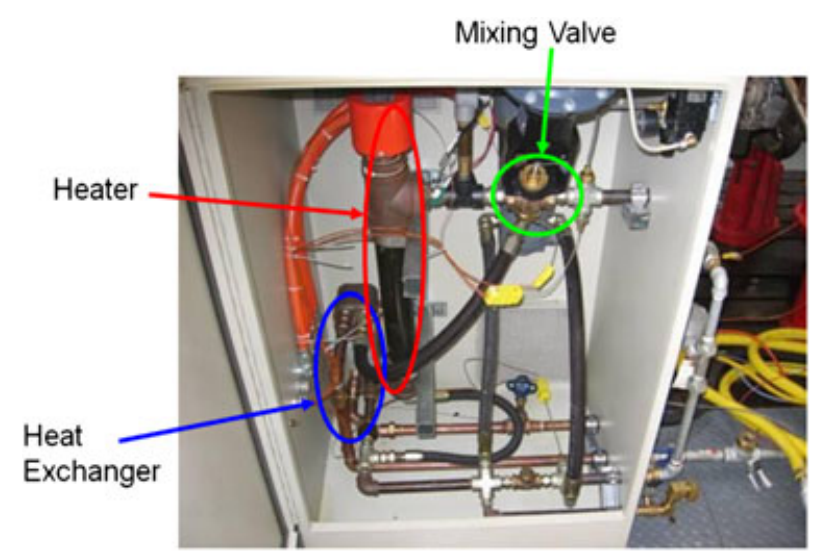

Figure 7. Oil fluid conditioning unit.

yielding the following modular system description:

$$
x_{1}=\left[\begin{array}{c}
\delta T_{\text {out,eng }} \\
\delta T_{\text {eng1 }} \\
\delta T_{\text {eng2 }}
\end{array}\right], x_{2}=\left[\begin{array}{c}
\delta T_{\text {out,mv }} \\
\delta T_{\text {out,ht }} \\
\delta T_{\text {ht }} \\
\delta T_{\text {out,hex }} \\
\delta T_{\text {out,cw }}
\end{array}\right], y=\delta T_{\text {out,eng }}, v=\delta T_{\text {out,mv }}, u=\delta \phi .
$$

For the linearized actuator dynamics, $A_{u}^{v}(s)$ is minimum phase, $\rho\left(A_{u}^{v}(s)\right)=1$, and $\rho\left(A_{x_{1}}^{v}(s)\right)=2$. Thus, our modeled actuator dynamics satisfy the conditions for equivalence between optimal modular and centralized system performance, making this system an excellent candidate for referencemodel-based modular control. Nevertheless, modeling uncertainties are present, and this will be the focal point of the MCEC design for the thermal management application.

\subsection{Inner-loop reference model}

For our system, we choose the simplest matchable reference model, namely

$$
F(s)=\frac{1}{\tau_{f} s+1} .
$$

In our system analysis and experimental results, we will explore the effect of adjusting $\tau_{f}$, both with and without MCEC in place.

\subsection{Outer-loop control design}

The objective of the outer-loop controller is to yield desirable overall system performance when the inner closed loop matches the reference model, that is, $V(s)=F(s) V_{\text {des }}(s)$. In our case, a simple outer-loop PI controller, given by

$$
\delta T_{\text {out }, \text { mv }}^{\text {des }}=\frac{2 s+0.05}{s}\left(\delta T_{\text {out,eng }}^{\text {des }}(s)-\delta T_{\text {out,eng }}(s)\right),
$$

yields excellent tracking performance when the reference model is matched.

\subsection{Inner-loop design}

Given our actuator model, it is possible to design the control law for $\delta \phi$ such that $\delta T_{\text {out,mv }}(s)=$ $F(s) \delta T_{\text {out,mv }}^{\text {des }}(s)$ (hence achieving perfect model matching) by taking

$$
\delta \phi=k_{1} \delta T_{\text {out,mv }}^{\text {des }}-k_{2} \delta T_{\text {out,mv }}-k_{3} \delta T_{\text {out,ht }}-k_{4} \delta T_{\text {out,hex }},
$$


where $\tau_{t}$ is the modeled time constant associated with the temperature dynamics of the mixing valve and $k_{1}, k_{2}, k_{3}$, and $k_{4}$ are directly determined from model parameters described in the detailed model in [27]. The values of $k_{1}, k_{2}, k_{3}$, and $k_{4}$ required for reference model matching are functions of the reference model time constant, $\tau_{f}$.

\subsection{Performance recovery in the presence of uncertainty — $\mu$ synthesis setup}

Given that our actuator model is not perfect, we now pursue an MCEC design that aims to keep the system within $10 \%$ of the ideal performance. Thus, we pursue synthesis objective S2 and take $\gamma^{\text {th }}=0.1$ to capture the $10 \%$ requirement.

To construct weighting functions, we consider three likely sources of uncertainty in the actuator model:

- Flow dynamics, where the flow distribution through the heater and the heat exchanger does not respond immediately, but rather through a time constant, $\tau_{\text {flow }}$;

- Uncertain time constant, $\tau_{t}$, associated with mixing valve thermal mixing dynamics;

- Inaccurate steady-state estimates, $\phi^{\mathrm{ss}}, T_{\mathrm{outht}}^{\mathrm{ss}}$, and $T_{\mathrm{outh}}^{\mathrm{ss}}$, which impact our linearized dynamics. We will see through experimental results that an uncertain value of $\phi^{\text {ss }}$ is especially realistic for two reasons. First, the valve is not position controlled (it is pressure controlled and sometimes yields inconsistent response). Second, the ambient temperature affects the required value of $\phi^{\text {ss }}$ to achieve a particular steady state (through the effect of ambient temperature on the cooling water temperature supplied to the system).

When augmented to include flow dynamics (33), the inner-loop dynamics that are affected by the uncertainty are given by

$$
\begin{gathered}
\delta \dot{\phi}=\frac{1}{\tau_{\text {flow }}}\left(-\delta \phi+\delta u_{\mathrm{mv}}\right), \\
\delta \dot{T}_{\text {out }, \mathrm{mv}}=\frac{1}{\tau_{t}}\left(-\delta T_{\text {out,mv }}+\phi^{\mathrm{ss}} \delta T_{\mathrm{out}, \mathrm{ht}}\right. \\
\left.+\left(1-\phi^{\mathrm{ss}}\right) \delta T_{\text {out,hex }}+\left(T_{\mathrm{out}, \mathrm{st}}^{\mathrm{ss}}-T_{\text {out,hex }}^{\mathrm{ss}}\right) \delta \phi\right),
\end{gathered}
$$

where the mixing valve command, $u_{\mathrm{mv}}$, does not immediately affect the valve position (and flow distribution), represented by $\phi$. The uncertain values, their nominal values, and their uncertain ranges are given in Table I.

We consider these aforementioned uncertainty sources to design the weighting transfer functions, $W_{1}(s)$ and $W_{2}(s)$, that are necessary for our $\mu$ synthesis design of $C_{v}$. Analyzing the closed-loop effects of these uncertainties through Bode plots of closed-loop systems generated by randomly varying uncertain parameters over there pre-specified range, we have found that the

Table I. Uncertain actuator model parameters.

\begin{tabular}{lcc}
\hline Parameter & Nominal value & Range \\
\hline$\tau_{\text {flow }}(\mathrm{s})$ & 0.00 & $0.0-2.0$ \\
$\tau_{t}(\mathrm{~s})$ & 8.00 & $6.0-10.0$ \\
$\phi^{\mathrm{ss}}$ & 0.75 & $0.7-0.8$ \\
$T_{\text {out,ht }}^{\mathrm{ss}}-T_{\text {out,hex }}^{\mathrm{ss}}\left({ }^{\circ} \mathrm{C}\right)$ & 40.80 & $35.0-45.0$ \\
\hline
\end{tabular}


following weighting functions capture the frequency-dependent impact of the expected sources of system uncertainty:

$$
\begin{aligned}
& W_{1}(s)=\frac{0.2(0.1 s+1)(100 s+1)}{(0.3 s+1)(20 s+1)}, \\
& W_{2}(s)=0.1
\end{aligned}
$$

Remark 13

Note that for the thermal management system, although there are three plant states, only one of these states has any effect on the actuator dynamics; thus, although $W_{2}(s)$ is a $3 \times 3$ transfer function matrix according to the formulation of (22), two of the entries in $W_{2}(s)$ (in (22)) will be equal to 0 . For simplicity, we drop these transfer functions from the formulation (the resulting $\Delta$ is $2 \times 2$ ).

\section{Remark 14}

Nonzero DC content is essential to capture the true system uncertainty because there is no integrator in the inner loop (nor is one necessary because of the outer-loop integrator), and uncertainties will contribute to a DC error.

$\mu$ synthesis was performed for several reference models (with different time constants, $\tau_{f}$ ), each leading to a different compensator, $C_{v}(s)$. In spite of the differences and the high orders of the resulting compensators, each reflects a somewhat de-tuned and causal approximation of the compensator $C_{v}(s)=1 / F(s)$, which results from setting $G^{e r}(s)=0$ and solving for $C_{v}(s)$. In light of this fact, the resulting compensators were parameterized as

$$
C_{v}(s)=\frac{b_{1} s+b_{0}}{a_{2} s^{2}+a_{1} s+a_{0}},
$$

where the transfer function coefficients were determined through a balanced truncation. The resulting worst-case performance levels, $\gamma^{\mathrm{wc}}$, under these (reduced-order) compensators, considering the aforementioned uncertainty sources, are given in Figure 8, for different values of $\tau_{f}$.

Figure 8 shows that although MCEC provides benefit for all reference models, it is most helpful for aggressive reference models (i.e., fast time constants), where the inner-loop designer is pursuing a higher-bandwidth reference model, which exacerbates the effect of uncertainties on inner-closedloop performance. Although the reference model is typically negotiated in the beginning stages of a control design project, the graph in Figure 8 can only be generated after the outer-loop controller

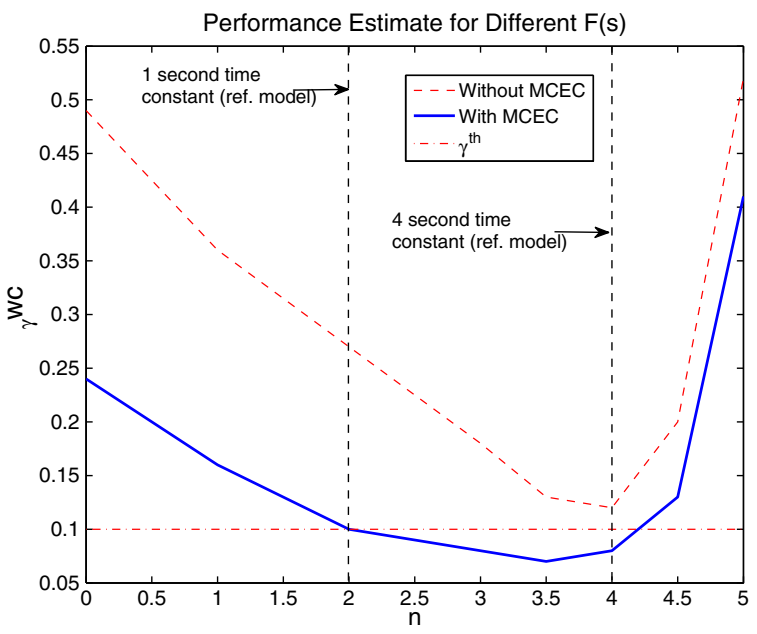

Figure 8. Performance comparison with and without modular control error compensation (MCEC), for different reference model time constants, based on speculated sources of uncertainty. In parameterizing the reference model, we take $\tau_{f}=0.25\left(2^{n}\right)$. 
has been designed and closed-inner-loop uncertainty has been characterized (through weighting functions). Therefore, although it is evident from Figure 8 that $F(s)=1 / 4 s+1$ represents a good reference model choice both with and without MCEC, this may not be known to a designer a priori; thus, it is advantageous to have MCEC available because it results in a system performance that is more robust to one's choice of reference model.

For very slow reference models, overall behavior degrades because the inner loop is designed to a bandwidth specification that is not well separated from that of the outer loop. This lack of bandwidth separation makes the overall system performance more sensitive to any mismatch between the reference model and actual inner-loop performance; thus, control performance both with and without MCEC deteriorates.

To gain deeper insight into the mechanisms by which MCEC improves performance, we can examine the Bode plots in Figures 9 and 10, which provide the open-loop transfer functions of the overall system (from where $\delta T_{\text {out }}^{\text {eng }}$ enters the outer-loop controller to where $\delta T_{\text {out }}^{\text {eng }}$ is outputted from the plant), under worst-case uncertain parameter values and for $\tau_{f}$ equal to 4 and $1 \mathrm{~s}$, respectively. The figures indicate improved phase margins with the use of MCEC, particularly for the case of $\tau_{f}=1 \mathrm{~s}$.

Whereas Figure 8 showed worst-case performance over a variety of reference models, Figures 11 and 12 show how worst-case performance varies with the level of uncertainty in system parameters. Specifically, Figures 11 and 12 provide worst-case performance levels when the parameters of Table I are allowed to vary over a specified percentage of their range (for example, for a value of $50 \%, \tau_{\text {flow }}$ may vary between 0 and $1 \mathrm{~s}, \tau_{t}$ may vary between 7 and $9 \mathrm{~s}$, and $\phi_{\mathrm{ss}}$ may vary between 0.725 and 0.775 ). To compare the use of MCEC with traditional applications of $\mu$ synthesis, Figures 11 and 12 additionally show the worst-case gains when MCEC is not used but instead the outer-loop controller, $C$, is designed using $\mu$ synthesis. From these figures, it is evident that although standard $\mu$ synthesis leads to improved performance in the presence of sufficiently large uncertainty, it also leads to a degradation in nominal performance, which is a trade-off that is not faced with MCEC.

\subsection{Experimental results}

The performance of the modular control strategy and MCEC was verified in an engine test cell, with the rapid prototyping setup using MATLAB Real-Time Workshop ${ }^{\mathrm{TM}}$ and $\mathrm{xPC}$ Target ${ }^{\mathrm{TM}}$. We consider temperature setpoint changes, where the engine is kept at constant speed and load (2000 rpm and $75 \mathrm{~N} / \mathrm{m}$, respectively). The plots given in Figures 13 and 14 show the data collected from

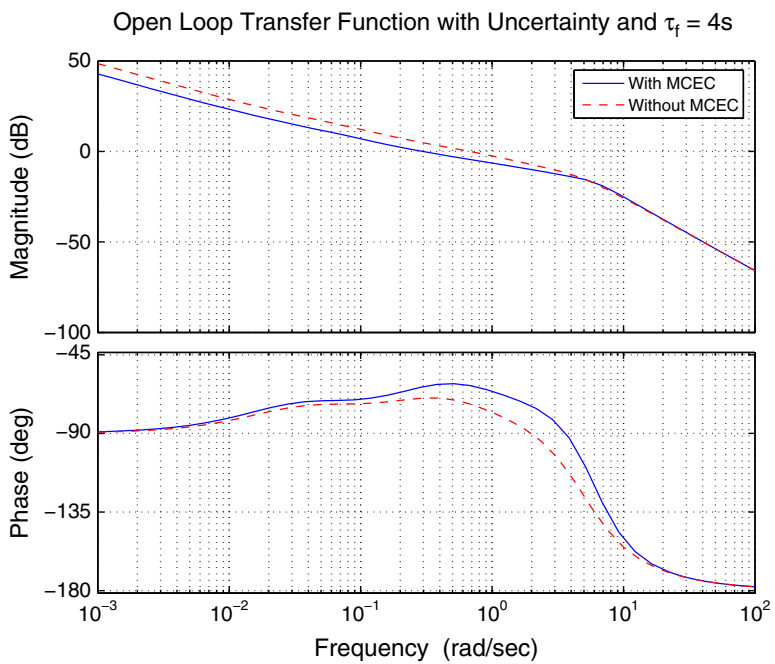

Figure 9. Open-loop transfer function under worst-case uncertain parameters with $\tau_{f}=4 \mathrm{~s}$. MCEC, modular control error compensation. 


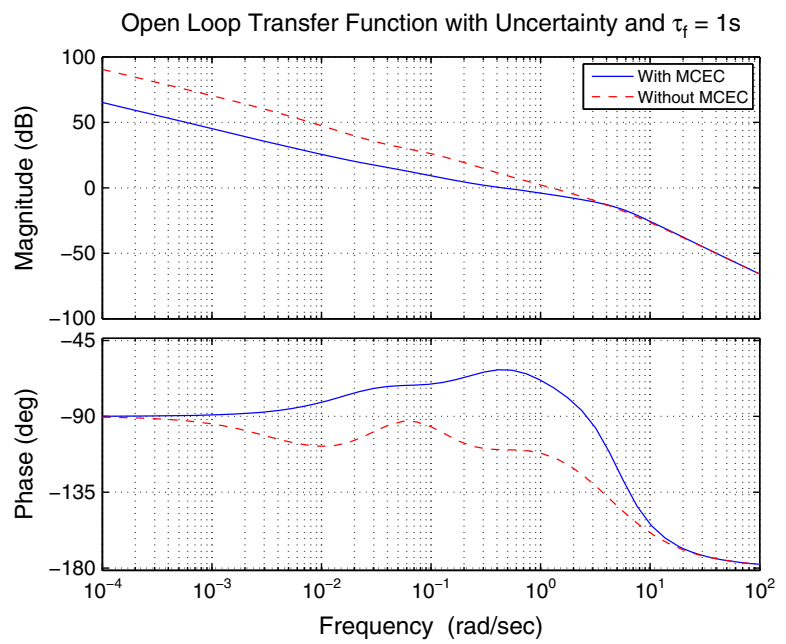

Figure 10. Open-loop transfer function under worst-case uncertain parameters with $\tau_{f}=1 \mathrm{~s}$. MCEC, modular control error compensation.

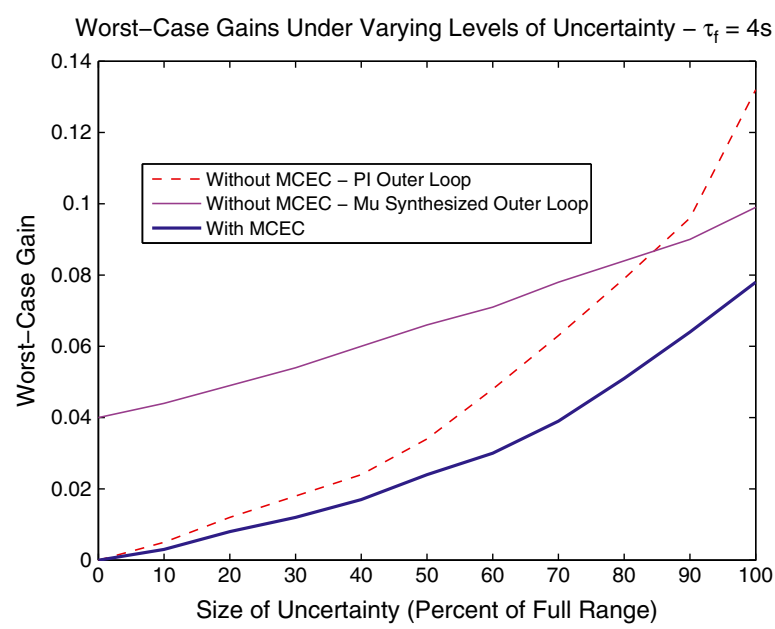

Figure 11. Worst-case performance under varied levels of uncertainty, for a reference model with $\tau_{f}=4 \mathrm{~s}$. MCEC, modular control error compensation.

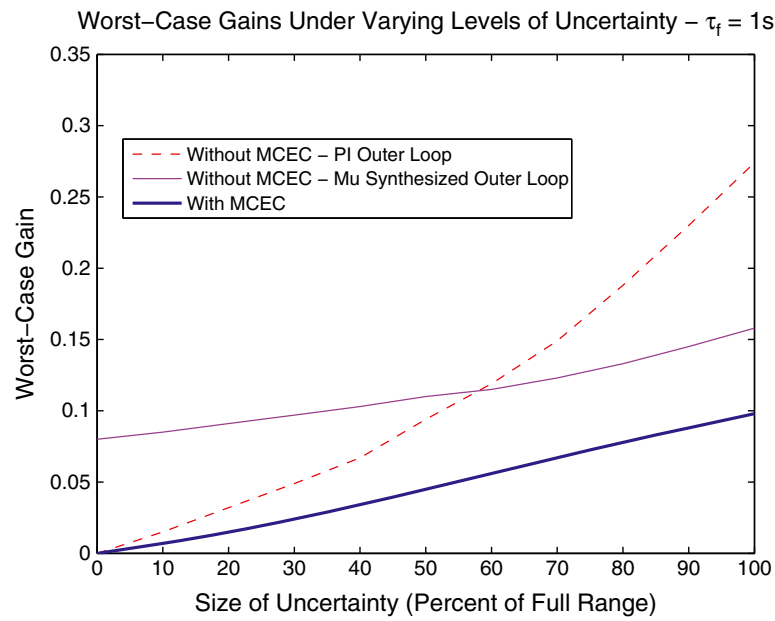

Figure 12. Worst-case performance under varied levels of uncertainty, for a reference model with $\tau_{f}=1 \mathrm{~s}$. MCEC, modular control error compensation. 

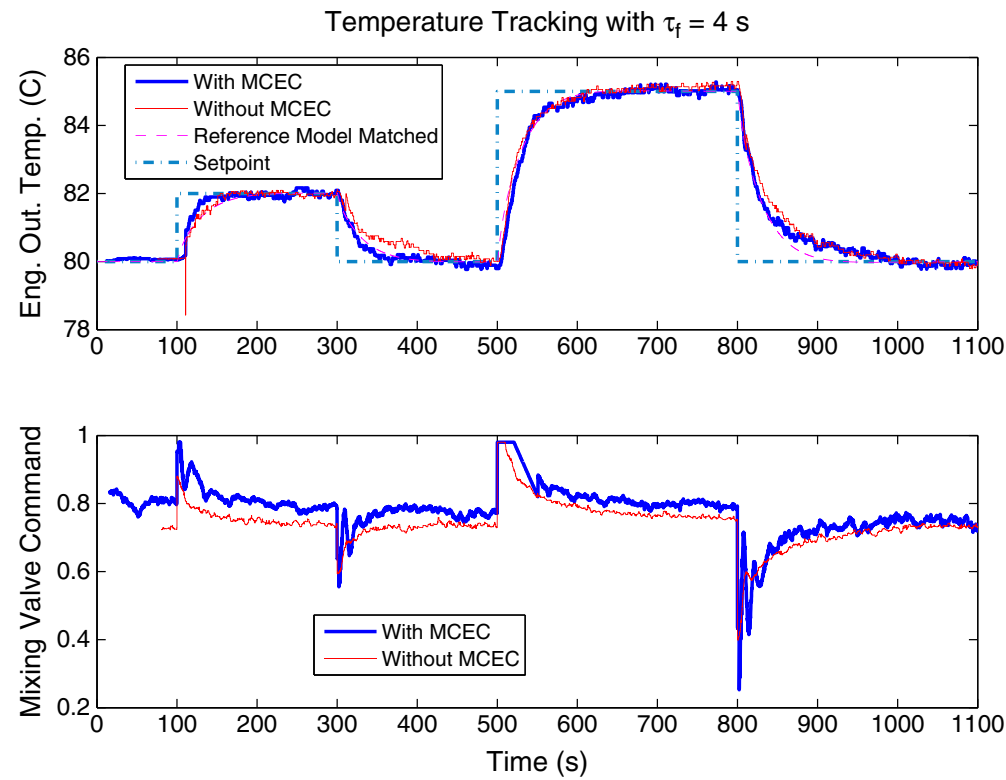

Figure 13. Experimental results with $\tau_{f}=4 \mathrm{~s}$. MCEC, modular control error compensation.
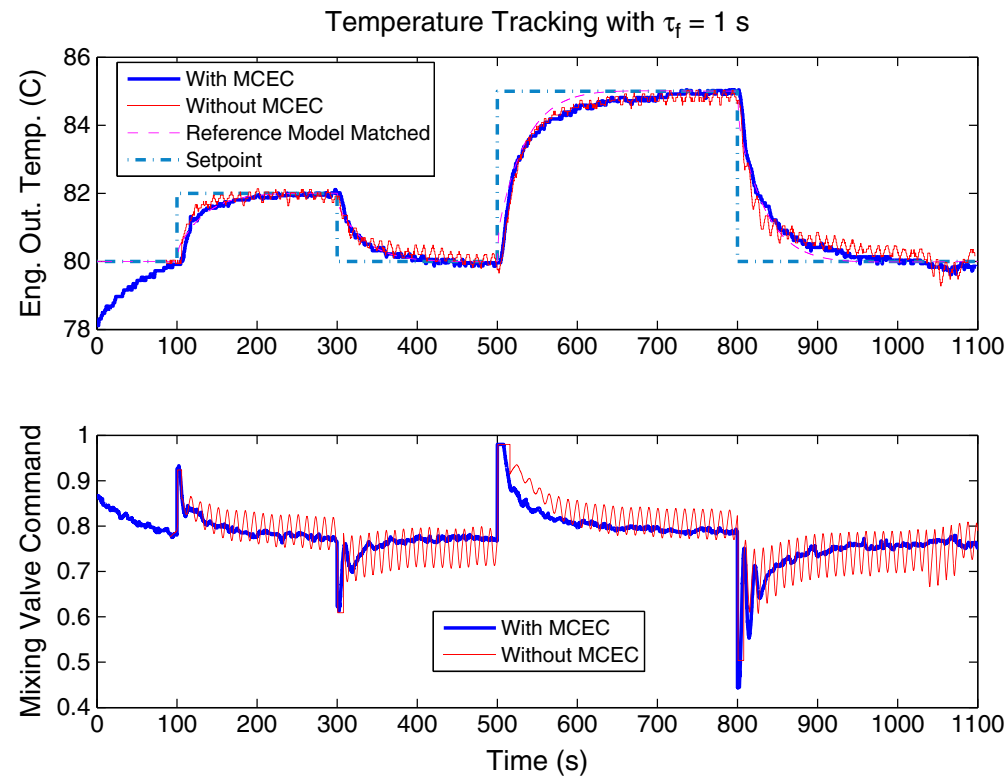

Figure 14. Experimental results with $\tau_{f}=1 \mathrm{~s}$. MCEC, modular control error compensation.

experiments, both with and without MCEC. The results are summarized, along with model-based analysis results, in Table II, where experimental results are characterized quantitatively by the performance metric

$$
\gamma^{\text {exper }}=\frac{\left(\sum_{i=0}^{n}\left(\left(\delta T_{\text {out,eng }}(i)-\delta T_{\text {out }, \text { eng }}^{r}(i)\right)^{2}\right)\right)^{1 / 2}}{\left(\sum_{i=0}^{n}\left(\left(\delta T_{\text {out }, \text { eng }}^{\text {des }}(i)\right)^{2}\right)\right)^{1 / 2}},
$$


Table II. Thermal management experimental and analysis results.

\begin{tabular}{lcccc}
\hline$\tau_{f}(\mathrm{~s})$ & $\gamma_{\text {MCEC }}^{\text {exper }}$ & $\gamma_{\text {No MCEC }}^{\text {exper }}$ & $\gamma_{\text {MCEC }}^{\text {wc }}$ & $\gamma_{\text {No MCEC }}^{\text {wc }}$ \\
\hline 1 & 0.074 & 0.129 & 0.098 & 0.274 \\
4 & 0.072 & 0.087 & 0.072 & 0.127 \\
\hline
\end{tabular}

MCEC, modular control error compensation.

where $\delta T_{\text {out,eng }}^{r}$ represents the engine outlet temperature (deviation from nominal) when the innerloop reference model is matched exactly. This metric is intended to approximate the gain from $r$ to $e^{\prime}$ for our experimental data.

As expected, $\gamma^{\text {exper }}$ is lower with MCEC than without under both reference models, but the difference is much more pronounced when $\tau_{f}=1$ (the more aggressive reference model). Also, with MCEC, $\gamma^{\text {wc }}<\gamma^{\text {th }}$ with both reference models, indicating that we have achieved acceptable performance with MCEC in both cases. For comparison, Table II also provides $\gamma^{\mathrm{wc}}$ as predicted through an analysis of hypothesized sources of uncertainty. The experimental results correlate directionally well with the worst-case analysis results.

\section{CONCLUSIONS AND FUTURE WORK}

In this paper, we have proposed a reference-model-based modular control design framework. We analyzed the nominal performance of a modular control system, concluding that under certain ideal circumstances, it can perform as well as its centralized counterpart. To treat cases when the inner closed loop does not perform exactly as specified, we have developed a modular control error compensator (MCEC) for recovering desired performance. We have shown how $\mu$ synthesis may be used iteratively to achieve the design goals and have demonstrated the effectiveness of the proposed method on an engine thermal management system. Future work will include the development of deeper insights with regard to the trade-offs between the inner-loop performance specification, $F$, and the ability to achieve robust modular performance with the use of $C_{v}$. Additionally, the ability to incorporate inherently nonlinear characteristics (especially saturation) into closed-loop uncertainty models that are applicable to MCEC represents a great avenue (and challenge) for future investigation.

\section{APPENDIX: PROOF OF PROPOSITION 4}

Sufficiency: Take $\rho_{1}=\rho\left(A_{u}^{v}(s)\right)$ and $\rho_{2}=\rho\left(A_{x_{1}}^{v}(s)\right)$, and express $F(s)$ as

$$
F(s)=\frac{N(s)}{D_{1}(s) D_{2}(s)},
$$

where $D_{2}(s)$ is $\rho_{1}^{\text {th }}$ order. Consider the control structure depicted in Figure A.15, which divides the controller into a feedback part that places $\rho_{1}$ poles and a feed-forward lead-lag element that places the zeros and remaining poles. The causality of the controller follows from condition (1) and Figure A.15, where $N(s) / D_{1}(s), K$, and $L$ are all proper.

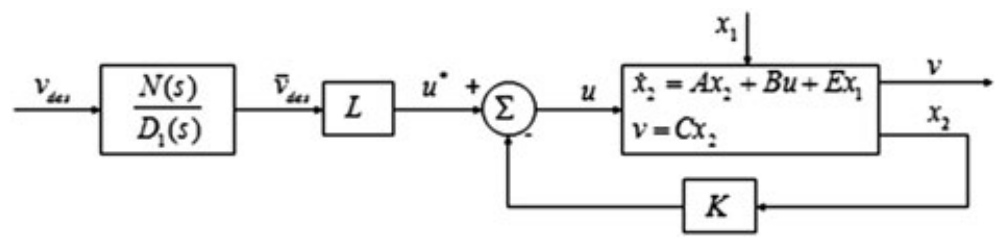

Figure A.15. Block diagram of the inner-loop controller used in this model-matching proof. 
Perform an observable realization of the actuator dynamics; $V(s)=A_{u}^{v}(s) U(s)+A_{x_{1}^{v}}(s) X_{1}(s)$ results in

$$
\begin{aligned}
{\left[\begin{array}{l}
\dot{\bar{x}}_{21} \\
\dot{\bar{x}}_{22}
\end{array}\right] } & =\left[\begin{array}{ll}
A_{11} & A_{12} \\
A_{21} & A_{22}
\end{array}\right]\left[\begin{array}{l}
\bar{x}_{21} \\
\bar{x}_{22}
\end{array}\right]+\left[\begin{array}{l}
B_{1} \\
B_{2}
\end{array}\right] u+\left[\begin{array}{l}
E_{1} \\
E_{2}
\end{array}\right] x_{1} \\
v & =\left[\begin{array}{llll}
1 & 0 & \ldots & 0
\end{array}\right] \bar{x}_{21},
\end{aligned}
$$

where $\bar{x}_{21} \in \mathbb{R}^{\rho_{1}}$ and $\bar{x}_{22} \in \mathbb{R}^{n_{2}-\rho_{1}}$,

$$
\begin{gathered}
B_{1}=\left[\begin{array}{ccc}
0_{\left(\rho_{1}-1\right) \times 1} & \ldots & 0_{\left(\rho_{1}-1\right) \times 1} \\
b_{1} & \ldots & b_{p}
\end{array}\right], \\
B_{2}=\left[\begin{array}{lll}
B_{21} & \ldots & B_{2 p}
\end{array}\right],
\end{gathered}
$$

where $B_{2 i} \in \mathbb{R}^{n-\rho_{1}}, \forall i$, and $b_{i}$ are scalars. From condition (2), $E_{1}=0$, which is essential for rejecting the $x_{1}$ disturbance. Define $u_{a} \triangleq u_{1}+\left(b_{2} / b_{1}\right) u_{2}+\ldots+\left(b_{p} / b_{1}\right) u_{p}, u_{b}=\left[\begin{array}{lll}u_{2} & \ldots & u_{p}\end{array}\right]^{\mathrm{T}}$ to obtain

$$
\begin{aligned}
{\left[\begin{array}{c}
\dot{\bar{x}}_{21} \\
\dot{\bar{x}}_{22}
\end{array}\right]=} & {\left[\begin{array}{ll}
A_{11} & A_{12} \\
A_{21} & A_{22}
\end{array}\right]\left[\begin{array}{c}
\bar{x}_{21} \\
\bar{x}_{22}
\end{array}\right] } \\
& +\left[\begin{array}{c}
B_{11} \\
B_{21}
\end{array}\right] u_{a}+\left[\begin{array}{c}
0_{\rho_{1} \times p-1} \\
\bar{B}_{22}
\end{array}\right] u_{b}+\left[\begin{array}{c}
0 \\
E_{2}
\end{array}\right] x_{1} \\
v= & {\left[\begin{array}{llll}
1 & 0 & \ldots & 0
\end{array}\right] \bar{x}_{21}, }
\end{aligned}
$$

where

$$
\begin{gathered}
B_{11}=\left[\begin{array}{ll}
0_{1 \times\left(\rho_{1}-1\right)} & b_{1}
\end{array}\right]^{\mathrm{T}} \\
\bar{B}_{22}=\left[\begin{array}{ccc}
B_{22}-\frac{b_{2}}{b_{1}} B_{21} & \ldots & B_{2 p}-\frac{b_{p}}{b_{1}} B_{21}
\end{array}\right] .
\end{gathered}
$$

Now consider the control law

$$
\begin{gathered}
u_{a}=L \bar{v}_{\mathrm{des}}-K_{11} \bar{x}_{21}-K_{12} \bar{x}_{22}, \\
u_{b}=-K_{22} \bar{x}_{22},
\end{gathered}
$$

where $K_{12}=\left[\begin{array}{llll}-1 / b_{1} & 0 & \ldots & 0\end{array}\right]$, which yields the following closed-loop dynamics:

$$
\begin{aligned}
{\left[\begin{array}{c}
\dot{\bar{x}}_{21} \\
\dot{\bar{x}}_{22}
\end{array}\right]=} & {\left[\begin{array}{cc}
A_{11}-\bar{B}_{11} K_{11} & 0_{\rho_{1} \times\left(n_{2}-\rho_{1}\right)} \\
A_{21}-\bar{B}_{21} K_{11} & A_{22}-\bar{B}_{22} K_{22}
\end{array}\right]\left[\begin{array}{c}
\bar{x}_{21} \\
\bar{x}_{22}
\end{array}\right] } \\
& +\left[\begin{array}{c}
B_{11} \\
B_{21}
\end{array}\right] L \bar{v}_{\mathrm{des}}+\left[\begin{array}{c}
0_{\rho_{1} \times p-1} \\
\bar{B}_{22}
\end{array}\right] u_{b}+\left[\begin{array}{c}
0 \\
E_{2}
\end{array}\right] x_{1} \\
v= & {\left[\begin{array}{llll}
1 & 0 & \ldots & 0
\end{array}\right] \bar{x}_{21} }
\end{aligned}
$$

The $\bar{x}_{21}$ dynamics are decoupled from the $\bar{x}_{22}$ dynamics (and hence from $x_{1}$ and $u_{b}$ ). It is easily verified that $\left(A_{11}, B_{11}\right)$ represents a controllable pair; hence, $K_{11}$ can be designed such that the eigenvalues of $A_{11}-B_{11} K_{11}$ are equal to those of $D_{2}(s)$. Let $M(s)$ represent the transfer function vector from $u^{*}$ to $v$ for fixed $K_{11}$ and $K_{12}$, where $M_{1}(s)$ represents the first element of $M(s)$, and take

$$
L=\left[\begin{array}{llll}
\frac{F(0) N(0)}{M_{1}(0) D_{1}(0)} & 0 & \ldots & 0
\end{array}\right]^{\mathrm{T}}
$$

to obtain $V(s)=F(s) V_{\text {des }}(s)$. 
We are left to use $u_{b}$ to stabilize the zero dynamics, given by

$$
\begin{aligned}
\dot{\bar{x}}_{22}= & \left(A_{22}-B_{21} K_{12}\right) \bar{x}_{22} \\
& +\bar{B}_{22} u_{b}+\left(A_{21}-B_{21} K_{11}\right) \bar{x}_{21}+E_{2} x_{1} .
\end{aligned}
$$

To do this, first note that the eigenvalues of $A_{22}-B_{21} K_{12}$ are identical to the zeros of $A_{u_{1}}^{v}(s)$. From condition (3), for every unstable zero, $\lambda_{i}$, of $A_{u_{1}}^{v}(s)$, there exists $j$, such that $\lambda_{i}$ is not a zero of $A_{u_{j}}^{v}(s)$ and is therefore also not a zero of $A_{u_{j}}^{v}(s)-\left(b_{j} / b_{1}\right) A_{u_{1}}^{v}(s)$. It follows that $\left(A_{22}-B_{21} K_{12}, \bar{B}_{22}\right)$ is a stabilizable pair, and therefore, there exists $K_{22}$ that stabilizes the zero dynamics of (A.12).

Necessity: To achieve $T_{1}(s)=F(s), T_{2}=0$, we require

$$
\begin{gathered}
A_{u}^{v}\left(I-C_{x_{2}}^{i} A_{u}^{x_{2}}\right)^{-1} C_{v_{\mathrm{des}}}^{i}=F, \\
A_{u}^{v}\left(I-C_{x_{2}}^{i} A_{u}^{x_{2}}\right)^{-1} C_{x_{2}}^{i} A_{x_{1}}^{x_{2}}=-A_{x_{1}}^{v} .
\end{gathered}
$$

The necessity of conditions (1) and (2) is obvious from comparing (A.13) and (A.14) with the following facts:

$$
\begin{gathered}
\rho\left(A_{u}^{v}\left(I-C_{x_{2}}^{i} A_{u}^{x_{2}}\right)^{-1} C_{v_{\text {des }}}^{i}\right) \geqslant \rho\left(A_{u}^{v}\right)>\rho(F), \\
\rho\left(A_{u}^{v}\left(I-C_{x_{2}}^{i} A_{u}^{x_{2}}\right)^{-1} C_{x_{2}}^{i} A_{x_{1}}^{x_{2}}\right)>\rho\left(A_{u}^{v}\right) \geqslant \rho\left(A_{x_{1}}^{v}\right) .
\end{gathered}
$$

Clearly, (A.13) and (A.14) cannot be achieved if the relative degrees of the left-hand and right-hand sides of (A.13) and (A.14) cannot even be made equal to each other.

Finally, suppose that there exists $\lambda$ such that $\operatorname{Re}(\lambda)>0$ and $A_{u_{j}}^{v}(\lambda)=0, \forall j$. Then, $A_{u}^{v}$ can be factored as $A_{u}^{v}=(s+\lambda) \bar{A}_{u}^{v}(s)$, and (A.13) can be written as

$$
(s+\lambda) \bar{A}_{u}^{v}\left(I-C_{x_{2}}^{i} A_{u}^{x_{2}}\right)^{-1} C_{v_{\mathrm{des}}}^{i}=F
$$

and for (A.15) to hold for any stable $F, \lambda$ must be canceled with an unstable pole (at $\lambda$ ), thereby destabilizing the actuator dynamics.

\section{ACKNOWLEDGEMENTS}

The authors wish to acknowledge Professors Le Yi Wang and Jim Freudenberg of the Electrical and Computer Engineering Department at Wayne State University and the Electrical Engineering and Computer Science Department at the University of Michigan, respectively, for discussions and insight regarding robust modular control results and $\mu$ synthesis. This work was supported by Toyota Motor Engineering and Manufacturing, North America (TEMA).

\section{REFERENCES}

1. Plumlee J, Bevly D, Hodel A. Control of a ground vehicle using quadratic programming based control allocation techniques. Proceedings of the American Control Conference, Boston, MA, 2004.

2. Chantranuwanthana S, Peng H. Adaptive robust control for active suspensions. Proceedings of the American Control Conference, San Diego, CA, 1999.

3. Harkegard O. Resolving actuator redundancy-control allocation vs. linear quadratic control. Proceedings of the European Control Conference, Cambridge, UK, 2003.

4. Bodson M. Evaluation of optimization methods for control allocation. Journal of Guidance, Control, and Dynamics 2002; 25(4):703-711.

5. Luo Y, Serrani A, Yurkovich S, Doman D, Oppenheimer M. Dynamic control allocation with asymptotic tracking of time-varying control input commands. Proceedings of the American Control Conference, Portland, OR, 2005.

6. Luo Y, Serrani A, Yurkovich S, Doman D, Oppenheimer M. Model Predictive dynamic control allocation with actuator dynamics. Proceedings of the American Control Conference, Boston, MA, 2004.

7. Luo Y, Serrani A, Yurkovich S, Oppenheimer M, Doman D. Model predictive dynamic control allocation scheme for reentry vehicles. Journal of Guidance, Control, and Dynamics 2007; 30(1):100-113. 
8. Lindeguard K, Fossen T. Fuel efficient rudder and propeller control allocation for marine craft: experiments with a model ship. IEEE Transactions on Control Systems Technology 2003; 11(6):850-862.

9. Johansen T, Fuglseth T, Tondel P, Fossen T. Optimal constrained control allocation in marine surface vessels with rudders. Control Engineering Practice 2008; 16(4):457-464.

10. Johansen T, Fossen T, Berge S. Constrained nonlinear control allocation with singularity avoidance using sequential quadratic programming. IEEE Transactions on Control Systems Technology 2004; 12(1):211-216.

11. Sordalen OJ. Optimal thrust allocation for marine vessels. Control Engineering Practice 1997; 5(9):1223-1231.

12. Tjonnas J, Johansen T. Optimizing adaptive control allocation with actuator dynamics. Proceedings of the IEEE Conference on Decision and Control, New Orleans, LA, 2007.

13. Kaya I. Improving performance using cascade control and a Smith predictor. ISA Transactions 2001; 40(3):223-234.

14. Lee Y, Park S. PID controller tuning to obtain desired closed loop responses for cascade control systems. Industrial \& Engineering Chemistry Research 1998; 35(5):1859-1865.

15. Sun J. A modified model reference adaptive control scheme for improved transient performance. IEEE Transactions on Automatic Control 1993; 38(8):1255-1259.

16. Vermillion C, Sun J, Butts K. Performance enhancement of modular control systems using $\mu$ synthesis. Proceedings of the IEEE Conference on Decision and Control, Cancun, Mexico, 2008.

17. Boulet B, Francis BA, Hughes PC, Hong T. $\mu$ synthesis for a large flexible space structure experimental testbed. Journal of Guidance, Control, and Dynamics 2001; 24.

18. Steinbuch M, Van Gross P, Schooststra G, Wortelboer P, Bosgra O. $\mu$ synthesis of a compact disc player. International Journal of Robust and Nonlinear Control 1998; 8:169-189.

19. Bibel J, Stalford H. $\mu$ synthesis autopilot design for a flexible missile. Aerospace Sciences Meeting, Reno, NV, 1991.

20. Adams R, Banda S. Robust flight control design using dynamic inversion and structured singular value synthesis. IEEE Transactions on Control Systems Technology 1993; 1:80-92.

21. Giusto A, Paganini F. Robust synthesis of feedforward compensators. IEEE Transactions on Automatic Control 1999; 44(8):1578-1582.

22. Fan M, Tits A. A measure of worst case $H_{\infty}$ performance and largest acceptable uncertainty. Systems and Control Letters 1992; 18(6):409-421.

23. Balas G, Doyle J, Glover K, Packard A, Smith R. $\mu$ Synthesis and Analysis Toolbox. The Math Works: Natick, MA, 2001.

24. Holland R, Young P, Zhu C. Development of a skew $\mu$ upper bound. Proceedings of the European Control Conference, 2003.

25. Ferreres G, Biannic J, Magni J. A skew $\mu$ toolbox for robustness analysis. Proceedings of the IEEE Symposium on Computer Aided Control Systems Design, Taipei, Taiwan, 2004.

26. Vermillion C, Sun J, Butts K, Hall A. Modeling and analysis of a thermal management system for engine calibration. Proceedings of the IEEE Conference on Control Applications, Munich, Germany, 2006.

27. Vermillion C. Optimal control of overactuated systems-theory and applications. PhD Dissertation, University of Michigan, 2009. 